\title{
Le travail dans l'économie des plateformes dans la jurisprudence italienne
}

\section{Vincenzo Bavaro et Donato Marino}

\section{(2) OpenEdition}

1 Journals

\section{Édition électronique}

URL : https://journals.openedition.org/rdctss/1548

DOI : 10.4000/rdctss. 1548

ISSN : 2262-9815

Éditeur

Centre de droit comparé du travail et de la sécurité sociale

\section{Édition imprimée}

Date de publication : 1 juin 2019

Pagination : 14-23

ISSN : 2117-4350

\section{Référence électronique}

Vincenzo Bavaro et Donato Marino, «Le travail dans l'économie des plateformes dans la jurisprudence italienne », Revue de droit comparé du travail et de la sécurité sociale [En ligne], 2 | 2019, mis en ligne le 01 novembre 2021, consulté le 11 novembre 2021. URL : http://journals.openedition.org/rdctss/1548 ; DOI : https://doi.org/10.4000/rdctss. 1548

\section{cc)}

Revue de droit comparé du travail et de la sécurité sociale est mise à disposition selon les termes de la Licence Creative Commons Attribution - Pas d'Utilisation Commerciale - Pas de Modification 4.0 International. 


\section{LE TRAVAIL DANS L'ÉCONOMIE DES PLATEFORMES DANS LA JURISPRUDENCE ITALIENNE'}
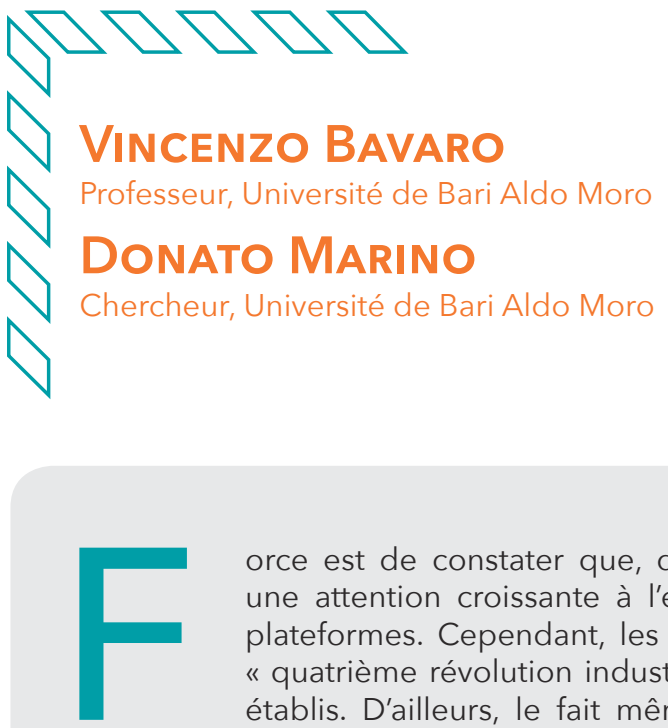

orce est de constater que, dans toute l'Europe, la doctrine porte une attention croissante à l'égard du travail dans l'économie des plateformes. Cependant, les caractéristiques et les effets de cette "quatrième révolution industrielle » ne sont pas encore clairement établis. D'ailleurs, le fait même que de multiples formules soient utilisées pour décrire cette phase de développement de l'économie capitaliste nous démontre la nécessité d'être encore plus attentifs dans la détermination des critères distinctifs des relations de travail instaurées au sein des entreprises du secteur de la production²: le chauffeur d'Uber, le coursier de Foodora, I'artisan de Victor, l'évaluateur d'AMT, effectuent des prestations de travail qui s'inscrivent dans le cadre de cet éventail de formes d'emplois.

La question des effets de l'innovation technologique sur les relations de travail est une antienne qui se répète périodiquement : songeons à la qualification de la relation de travail des personnels externes employés dans les centres d'appel, ou

1 Le contenu de l'article est le fait des deux auteurs. Cependant, D. Marino a rédigé les parties 2 et 3 , tandis que $V$. Bavaro a rédigé les parties 1 et 4 .

2 Gig economy, sharing economy, platform economy, etc... Ces différentes formules démontrent l'hétérogénéité des caractères distinctifs de cette nouvelle "économie ". Pour les auteurs, il s'agit d'un « modèle d'organisation de l'entreprise et du travail qui, en ayant recours aux technologies informatiques et à la possibilité d'être perpétuellement connecté à internet, propose, face aux demandes qui se présentent au fur et à mesure, des produits, des services et des compétences " (G.A. Recchia, " Gig economy e dilemmi qualificatori: la prima sentenza italiana », il Lavoro nella Giurisprudenza, 2018, $n^{\circ} 7$, p.726). 
au travail dans les systèmes d'organisation ayant adopté le modèle de ce que l'on a dénommé la " qualité totale ${ }^{3}$ ", ou encore plus tôt, aux pony express.

C'est dans ce contexte que le juge italien est aujourd'hui appelé à se prononcer sur le type spécifique des contrats de travail des coursiers à vélo. Les modalités d'exécution de cette relation de travail - caractérisée par l'intermédiation d'une plateforme informatique dans l'organisation du travail - remémore en Italie l'affaire des Pony express dans la jurisprudence des années $80^{4}$.

Le problème pour les juristes spécialistes du droit du travail vient du fait que ces contrats de travail sont tous, du point de vue formel, de type «indépendant», et donc dépourvus des protections garanties par le droit du travail aux travailleurs subordonnés. Tout cela parce que, selon une représentation de la doctrine, la plateforme de travail transformerait le travail subordonné en travail indépendant ${ }^{5}$. Or, tous les travailleurs des plateformes numériques aspirent à bénéficier des protections du travail subordonné, qu'il s'agisse des chauffeurs de la plateforme FedEx (dans l'Oregon et en Californie), d'Uber (en Californie et en Angleterre ${ }^{6}$ ), de Lyft (en Californie), du travailleur de CrowdFlower, ou encore des coursiers à vélo de Foodora ou Foodinho en Italie. Toutes ces personnes ont sollicité auprès de leurs tribunaux nationaux respectifs la protection juridique accordée aux travailleurs salariés ${ }^{7}$, obligeant systématiquement à soulever la question théorique récurrente de la qualification juridique des relations de travail instaurées avec les travailleurs app-driven ${ }^{8}$ : sont-ils des travailleurs subordonnés ou des indépendants?

3 L. Gaeta, «Qualità totale e teorie della subordinazione », Diritto delle Relazioni Industriali, 1994, p. 3, et L. Castelvetri, «Contratto di lavoro e qualità totale », Rivista Giuridica del Lavoro, 1994, I, p. 431.

4 R. Voza, dans " II lavoro e le piattaforme digitali: the same old story ? ", Working Paper Massimo D'Antona, n' 336/2017, a raison de dire qu'il n'y a pas de grande différence, du point de vue de la qualification de la relation de travail, entre le coursier de Foodora et le coursier de l'affaire Pony express auquel la doctrine juridique italienne du milieu des années 80 a prêté attention. Voir le jugement du Tribunal d'instance de Milan du 20 juin 1986, in Orientamenti di Giurisprudenza del Lavoro, 1986, p.978, suivi d'une note de L. Spagnuolo Vigorita, «Subordinazione e impresa ».

5 P. Ichino, "Le conseguenze dell'innovazione tecnologica sul diritto del lavoro ", Rivista Italiana di Diritto del Lavoro, 2017, I, p. 525.

6 A. Donini, «Regole della concorrenza e attività di lavoro nella on demand economy: brevi riflessioni sulla vicenda Uber », Rivista Giuridica del Lavoro, 2016, II, p. 46.

7 Ce n'est pas un hasard si les toutes les actions en justice sont des recours contre un licenciement ou sont relatives à des demandes de salaire ; cf. A. Donini, « Il lavoro su piattaforma digitale "prende forma" tra autonomia e subordinazione. Nuove regole per nuovi lavori? ", Diritto delle Relazioni Industriali, 2016, p. 174-175.

8 P. Tullini, «Prime riflessioni dopo la sentenza di Torino sul caso Foodora », Lavoro Diritti Europa, 2018 , p. 1 ; C. Spinelli, « La qualificazione giuridica del rapporto di lavoro dei fattorini di Foodora tra autonomia e subordinazione », Rivista Giuridica del Lavoro, 2018, II, n³ 3, p. 371. 


\section{I - PREMIÈRE THÈSE : LA RELATION DE TRAVAIL DES COURSIERS N'EST PAS SUBORDONNÉE}

La question de la qualification juridique de la relation de travail des coursiers aboutit, pour la première fois devant les instances judiciaires italiennes, à la suite du recours présenté par des travailleurs engagés par la société Foodora et par la société Foodinho sur la base de contrats de travail non subordonné, mais de «collaboration coordonnée et continue».

Jusqu'à présent, les affaires judiciaires italiennes ne sont qu'au nombre de deux et ont fait l'objet de trois décisions : le Tribunal ${ }^{9}$ puis la Cour d'appel ${ }^{10}$ de Turin dans une affaire de six travailleurs engagés en vertu de contrats de collaboration coordonnée et continue - au titre de l'art. 409 du Code de procédure civile - pour livrer à vélo des plats cuisinés pour le compte de la société Foodora et enfin le Tribunal de Milan ${ }^{11}$ pour le cas d'un travailleur engagé au titre d'un contrat de collaboration coordonnée et continue - qui effectuait des livraisons avec son propre véhicule pour le compte de la société Foodinho (qui intervient en Italie via la plateforme espagnole Glovo). Dans les deux affaires, les coursiers ont demandé que leur relation de collaboration coordonnée et continue soit qualifiée de relation de travail subordonnée.

L'examen des modalités d'exercice de l'activité professionnelle des coursiers a conduit les tribunaux, tout comme la Cour d'appel, à rejeter les demandes des travailleurs et à déclarer qu'il n'y avait là aucune relation de travail subordonnée dans la mesure où les travailleurs n'étaient assujettis, ni au pouvoir de direction et d'organisation de leur employeur, ni à son pouvoir disciplinaire. Ces tribunaux ont réaffirmé une orientation de la Cour de cassation selon laquelle la subordination comporte une « obligation de soumission du travailleur au pouvoir de direction, d'organisation et de sanction de l'employeur, qui découle de l'émission d'ordres spécifiques, ainsi que de l'exercice d'une activité assidue de surveillance et de contrôle de l'exécution des prestations de travail $»^{12}$.

Les tribunaux ont accordé de l'importance au fait que dans tous les contrats de collaboration, il existe une clause dite "independent contractor » à travers laquelle on a voulu mettre l'accent sur l'indépendance du coursier appelé à effectuer sa prestation « en toute indépendance, sans être assujetti à un quelconque lien de subordination, pouvoir hiérarchique ou disciplinaire, ou encore à des obligations de présence ou des contraintes horaires de tout type envers le donneur d'ordre».

9 Tribunal de Turin, 07.05.2018. P. Ichino, «Subordinazione, autonomia e protezione del lavoro nella Gig-Economy», Rivista Italiana di Diritto del Lavoro, 2018, II, p. 294 ; M. Del Conte, O. Razzolini, « La gig economy alla prova del giudice : la difficile reinterpretazione della fattispecie e degli indici denotativi », Giornale di Diritto del Lavoro e di Relazioni Industriali, 2018, n³, p. 673.

10 Cour d'appel de Turin, 04.02.2019.

11 Tribunal de Milan, 10.9.2018; C. Spinelli, «Rider: anche il Tribunale di Milano esclude il vincolo di subordinazione nel rapporto lavorativo », Rivista Giuridica del Lavoro, 2019, nº 1.

12 Les décisions des tribunaux font expressément référence à la Cour de Cassation (arrêt $n^{\circ} 2728$ du 08.02.2010 et arrêt $n^{\circ} 21028$ du 28.09.2006). 
Cependant, tout en tenant compte de la volonté des parties exprimée à travers cette clause, les tribunaux ont jugé bon de vérifier la nature indépendante ou subordonnée de la relation de travail, au moyen d'un examen des modalités concrètes d'exécution de la prestation de travail et en faisant abstraction de la qualification juridique donnée au contrat par les parties ${ }^{13}$.

Pour ce qui est de la qualification juridique de la relation de travail, l'élément déterminant résidait dans l'importance accordée par les tribunaux à la liberté du coursier de déterminer s'il devait effectuer la livraison, quand l'effectuer et pendant combien de temps être disponible. En effet, la relation de travail était caractérisée par le fait, d'une part que les coursiers n'avaient aucune obligation d'effectuer la prestation de travail et, d'autre part que l'employeur n'était nullement obligé de recourir à leur disponibilité pour effectuer des livraisons. Cette «liberté » contractuelle dans la gestion de la relation a concerné tout autant le travail des coursiers de Foodora que ceux de Foodinho.

La relation de travail était gérée au moyen d'une plateforme multimédia (appli) sur laquelle la société publiait une fois par semaine les tours de service (slots), en indiquant le nombre de coursiers requis pour couvrir chaque tour de service. Chaque coursier pouvait donc se montrer disposé à assurer les différents slots en fonction de ses exigences personnelles, mais il n'était pas obligé de le faire. Une fois reçue confirmation de l'affectation de son tour de service de la part du « responsable du parc », le travailleur devait se rendre dans l'une des zones de départ préétablies, se connecter sur l'appli et activer la géolocalisation. À ce moment-là, le coursier recevait sur l'appli la notification de la commande avec l'indication du restaurant et, une fois la commande acceptée, il devait prendre en charge les produits en utilisant son propre véhicule (vélo ou voiture), vérifier qu'ils correspondaient bien à la commande et, après avoir chargé les plats dans le coffre, veiller à les livrer au client dans un délai donné.

Le coursier pouvait par conséquent se rendre disponible pour effectuer l'un des tours de service indiqués par Foodora ou par Foodinho, mais sans être obligé de le faire ; de la même façon, I'appli pouvait également ne pas accepter la disponibilité exprimée par un travailleur et ne pas l'inclure dans les tours de service.

Selon le Tribunal de Turin, cette caractéristique de la relation « est en soi déterminante pour exclure la soumission des demandeurs au pouvoir de direction et d'organisation de l'employeur. En effet, il est évident que si l'employeur ne peut exiger du travailleur qu'il exécute la prestation de travail, il ne peut d'autant pas exercer sur lui son pouvoir de direction et d'organisation ». De manière analogue, le Tribunal de Milan a également vu dans ce « facteur essentiel de l'autonomie organisationnelle, se traduisant par la liberté de fixer la quantité et le moment de la réalisation de la prestation de travail [...] un élément incompatible avec le lien de subordination ». Selon le Tribunal de Milan, la subordination implique «l'intégration du travailleur dans l'organisation de l'entreprise de l'employeur, en

13 Pour la jurisprudence italienne, « aux fins de la détermination de la nature indépendante ou subordonnée d'une relation de travail, la qualification formelle établie par les parties au moment de la conclusion du contrat individuel, même si elle est significative, n'est pas déterminante, étant donné que les parties, tout en souhaitant mettre en œuvre une relation de travail subordonnée, pourraient très bien avoir simultanément déclaré souhaiter une relation indépendante en vue de contourner la réglementation légale en la matière " (Cour de cassation, arrêt n 19199 du 19.08.2013; Cour de cassation, arrêt n 7024 du 08.04.2015; Cour de cassation, arrêt n 4884 du 01.03.2018). 
contrepartie de la mise à disposition de ses capacités de travail (operæ) au bénéfice de celui-ci ainsi que de sa soumission concomitante au pouvoir de direction de ce dernier", alors que « dans le travail indépendant, l'objet de la prestation est constitué par le résultat de l'activité (opus)».

Par conséquent, pour les tribunaux de Turin et Milan, étant donné que l'obligation d'exécution de la prestation de travail fait défaut - au sens où il n'y a aucune obligation d'être disposé à s'intégrer dans un tour de service (slot) -, la possibilité de soumission au pouvoir de direction, d'organisation et de sanction de la société est totalement absente ; l'existence d'un lien de subordination est donc exclue d'emblée. L'absence de risque économique, la détermination du lieu de la prestation, le mode de rémunération sont des indices qui «ne peuvent avoir qu'une valeur indicative et non déterminante, ces éléments ne constituant par eux-mêmes que des facteurs qui, bien que significatifs dans la reconstitution de la relation, sont susceptibles in abstracto d'être compatibles avec l'une comme l'autre des qualifications de la relation en question ${ }^{14}$.

Néanmoins, comme la société peut exercer son pouvoir de direction et d'organisation, dès l'instant où les coursiers sont disposés à travailler et ensuite intégrés dans un tour de service, les tribunaux ont vérifié dans quelle mesure, pendant les tours de service des coursiers, les sociétés ont effectivement exercé leur pouvoir de direction et d'organisation. Cette seconde analyse des modalités concrètes du déroulement de la prestation a renforcé la conviction des tribunaux sur l'inexistence d'un pouvoir de direction sur les coursiers.

Selon les tribunaux de Turin et Milan, tout en admettant qu'à l'ére du travail numérique, ce que l'on appelle l'hétéro-direction - élément caractéristique de la subordination, qui consiste dans "l'émission d'ordres spécifiques et dans l'exercice d'une activité assidue de surveillance et de contrôle de l'exécution des prestations de travail $»^{15}$ - pourrait être exercée au moyen d'instruments faisant abstraction de la présence physique, en un seul et même lieu, de la personne qui donne les ordres et de celle qui les reçoit, cette hétérodirection n'ayant été exercée par aucune des sociétés, celles-ci s'étant limitées à l'exercice d'un simple pouvoir de coordination des prestations, typique des relations de travail indépendantes.

La détermination du lieu et des heures de travail (points de départ et créneaux horaires), le contrôle de la présence des coursiers aux points de départ, le rappel des travailleurs qui tardent à accepter la commande, la nécessité - en cas d'impossibilité d'effectuer la prestation de travail - de transmettre à ses supérieurs une demande de réaffectation de la commande, l'obligation d'effectuer la livraison des produits dans des délais préétablis en suivant le trajet suggéré par le GPS de l'appli, le suivi de la productivité de chacun des travailleurs... Rien de tout cela, aux dires des tribunaux, n'a pu être constaté dans les éléments de la procédure et, de toute manière, il s'agissait là d'indices jugés non déterminants pour la qualification de la relation de travail subordonnée. Selon les tribunaux, les instructions fournies par les sociétés sur le contenu et les objectifs de la mission confiée au cas par cas, ainsi que la fixation de normes qualitatives et quantitatives relatives aux prestations, constituaient l'exercice d'une activité de coordination du donneur d'ordre typique de la relation de travail indépendante.

14 En ce sens, voir l'arrêt de la Cour de cassation nº 15631 du 14.06.2018.

15 Tel qu'écrit par le Tribunal de Turin le 07.05.2018 et rappelé dans la jurisprudence de la Cour de cassation. 
Par ailleurs, dans le cas Foodora, le tribunal a également estimé qu'il n'y avait pas lieu de mettre en avant la soumission des travailleurs au pouvoir disciplinaire, c'est-à-dire à l'avertissement verbal et à l'exclusion temporaire ou définitive du forum de discussion (chat) de l'entreprise ou des tours de service. Selon le Tribunal de Turin, dès lors que les coursiers se sont vu attribuer le pouvoir d'annuler leur disponibilité sur un tour de service déjà confirmé par la société en utilisant la fonction de permutation (dite swap), voire de ne pas effectuer la prestation sans aucune communication préalable (fonction dite no show), et que l'exercice de ce pouvoir par les coursiers n'a jamais entraîné une quelconque sanction disciplinaire, cela confirme bien leur absence de subordination. L'avertissement verbal et l'exclusion temporaire ou définitive du forum de discussion de l'entreprise ou des tours de service n'ont pas été considérés comme l'exercice d'un pouvoir disciplinaire car cela n'avait pas pour objet de «priver les travailleurs d'un de leurs droits». En définitive, les Tribunaux de Turin et de Milan, ainsi que la Cour d'appel de Turin, ont déclaré que les relations de travail des coursiers n'étaient pas subordonnées au titre de l'art. 2094 du Code civil.

\section{II - DEUXIÈME THÈSE : LA RELATION DE TRAVAIL DES COURSIERS EST UNE COLLABORATION HÉTÉRO-ORGANISÉE}

Dans les trois décisions, les tribunaux italiens ont soulevé une deuxième question. Après avoir repoussé la demande des coursiers relative au constat de leur subordination, ces derniers se sont également penchés sur l'éventualité que les relations de travail en question puissent être qualifiées de relations de travail hétero-organisé, conformément à l'art. 2 du décret-loi n 81/2015.

Cette règle de droit établit qu'« à compter du 1er janvier 2016, la réglementation de la relation de travail subordonnée s'applique aussi aux relations de collaboration qui se concrétisent par des prestations de travail exclusivement personnelles, continues et dont les modalités d'exécution sont organisées par le donneur d'ordre, notamment en ce qui concerne le temps et le lieu de travail ». Cette règle a suscité un vif débat dans la doctrine italienne ${ }^{16,}$ qui s'est reflété dans les trois décisions examinées. Cette fois cependant, les tribunaux ont tranché différemment.

Pour le Tribunal de Turin, cette règle n'aurait aucun « contenu susceptible de produire de nouveaux effets juridiques sur le plan de la réglementation applicable aux différents types de relations de travail ». Ce tribunal a adhéré à la doctrine soutenant que l'art. 2 du décret législatif $n^{\circ} 81 / 2015$ est une "règle apparente $~^{17}$ voire " inutile ${ }^{18}$, c'est-àdire "se limitant à inscrire dans le droit positif une orientation extensive antérieure de la jurisprudence ${ }^{19}$. En d'autres termes, selon cette doctrine, la « collaboration continue

16 S. Ciucciovino, "Le collaborazioni organizzate dal committente nel confine tra autonomia e subordinazione», Rivista Italiana di Diritto del Lavoro, 2016, I, p. 321; G. Ferraro, "Collaborazioni organizzate dal committente », Rivista Italiana di Diritto del Lavoro, 2016, I, p. 53.

17 P. Tosi, "L'art. 2, comma 1, d. Igs. n. 81/2015 : una norma apparente ? ", Argomenti di Diritto del Lavoro, 2015, n 6, p. 1117; C. Pisani, « Le collaborazioni coordinate e continuative a rischio estinzione », Rivista Italiana di Diritto del Lavoro, 2018, I, p. 43.

18 C. Salazar, "Diritti e algoritmi : la Gig Economy e il "caso Foodora", tra giudici e legislatore », Consulta Online, 2019, fasc. 1, p. 154.

19 M. Biasi, "L'inquadramento giuridico dei riders alla prova della giurisprudenza », Lavoro Diritti Europa, 2018, n², p. 9. 
organisée par le donneur d'ordre » (hétéro-organisée) n'est rien d'autre qu'une manière différente d'entendre la collaboration subordonnée prévue par l'art. 2094 du Code civil. Cela serait d'autant plus vrai que la loi, en cas de collaboration hétéro-organisée, attribue les mêmes protections que celles prévues pour le travail subordonné. En conséquence, après avoir exclu la subordination, le Tribunal de Turin a également écarté la collaboration hétéro-organisée.

Ce faisant, on finit assurément par introduire une notion de travail subordonné encore plus restreinte que celle contenue dans l'art. 2094 du Code civil, dans la mesure où le travailleur devrait être assujetti au pouvoir de direction de l'employeur, lequel devrait se concrétiser encore et toujours par rapport au temps et au lieu de travail.

Le Tribunal de Milan aboutit de surcroît à la même conclusion, quoique de manière différente. Dans ce cas, l'art. 2 du décret législatif n 81/2015 n'apparaît pas tant comme une règle « apparente » que comme une règle qui « aurait uniquement pour effet d'étendre à certains travaux indépendants se distinguant par certaines de leurs caractéristiques de faiblesse économique et/ou organisationnelle, voire par une certaine proximité "morphologique" par rapport au travail subordonné, la réglementation de celui-ci ». Compte tenu des modalités d'exécution de la prestation, le Tribunal de Milan n'a pas estimé devoir qualifier la relation de travail de relation hétéro-organisée, "étant donné que le choix fondamental concernant les temps de travail et de repos a été laissé à la discrétion du demandeur, qui l'exerçait au moment où il faisait savoir qu'il était disposé à travailler certains jours et à certaines heures, et non pas à d'autres ». Comme on peut le voir, le fait que le coursier ait la liberté de choisir s'il doit travailler ou non est, une fois de plus, déterminant.

Toutefois, comme le tribunal ne saurait ignorer le fait qu'une fois intégré dans les tours de service, le travailleur est intégré dans l'organisation en recevant des directives, il se soucie de préciser que «le fait que, dans l'exécution concrète de son activité, le demandeur soit tenu, après avoir accepté d'effectuer une livraison, de finaliser celle-ci le plus rapidement possible n'est pas de nature à inclure le paramètre susmentionné, car il ne semble pas que la demande, par le donneur d'ordre, d'exécuter le travail dans un délai déterminé puisse être considérée comme une organisation du temps de travail ».

La décision de la Cour d'appel de Turin est différente ${ }^{20}$. La Cour, tout en convenant de l'inexistence de la subordination, a en effet estimé que l'art. 2 du décret-loi n 81/2015 a instauré un nouveau cas de contrat de collaboration hétéro-organisée, permettant de définir une troisième catégorie de contrat entre la relation de travail subordonnée au titre de l'art. 2094 du Code civil et la collaboration coordonnée et continue au titre de l'art. 409 n. 3 du Code de procédure civile. Le nouveau cas de contrat introduit par la loi, ayant un « contenu normatif », vise à " assurer une meilleure protection des nouvelles formes de travail qui, suite à l'évolution des technologies les plus récentes et à leur mise en œuvre toujours plus rapide, sont en train de se développer ». Donc, la collaboration est hétéroorganisée lorsque "l'on constate une intégration fonctionnelle effective du travailleur dans l'organisation de la production du donneur d'ordre, de sorte que la prestation de travail finit par être structurellement liée à celle-ci (l'organisation) et se présente comme quelque chose allant au-delà de la simple coordination visée à l'art. 409 alinéa 3 du Code

20 U. Carabelli et C. Spinelli, « La Corte d'Appello di Torino ribalta il verdetto di primo grado : i rider sono collaboratori etero-organizzati », Rivista Giuridica del Lavoro, 2019, nº 1. 
de procédure civile, car dans ce cas, c'est le donneur d'ordre qui détermine les modalités de l'activité de travail exercée par son collaborateur ».

En définitive, compte tenu des modalités d'exécution concrètes de la prestation de travail, la Cour d'appel de Turin a jugé bon de qualifier la relation de travail de collaboration hétéro-organisée en précisant que « le travailleur hétéro-organisé reste du point de vue technique 'indépendant' mais pour tout le reste, notamment en ce qui concerne la sécurité et l'hygiène, la rémunération directe ou différée, les limitations d'horaires, les congés et la sécurité sociale, la relation est régie » comme si elle était de nature subordonnée.

Ce qu'il convient de mettre en évidence, suite à l'arrêt de la Cour d'appel de Turin, c'est que les tribunaux excluent qu'il puisse s'agir là d'un travail subordonné. En effet, la relation de travail est fondée sur une double liberté du travailleur : d'une part, la liberté de s'intégrer ou non dans les tours de service, à savoir la liberté de se mettre à la disposition de l'organisation, d'autre part, une fois intégré dans ces tours, la liberté d'accepter ou non les appels de livraison.

La seule possibilité permettant de garantir des protections à ces relations de travail est d'admettre l'existence d'une hétéro-organisation en se référant au temps et au lieu de la prestation de travail, laquelle - selon la Cour d'appel de Turin - existe chaque fois que le coursier accepte d'effectuer une livraison. C'est pour cette raison que les coursiers de Foodora ont obtenu le droit aux mêmes protections que les travailleurs subordonnés dans la limite du temps d'exécution effective de la prestation de travail. Autrement dit, la liberté par rapport à l'obligation existe également lorsque le coursier est disposé à s'intégrer dans un tour de service ; mais chaque fois qu'il est appelé pour une livraison, une relation de travail organisée par la société se met aussi en place, y compris en ce qui concerne le temps et le lieu.

\section{III - LA QUESTION (NON RÉSOLUE) DE LA DIMENSION TEMPORELLE DU TRAVAIL SUBORDONNÉ}

En conclusion, nous estimons être en mesure d'affirmer que la question essentielle relative à la qualification de la relation de travail gérée par des plateformes informatiques (et donc, les protections qui doivent être garanties à ces travailleurs) réside précisément dans la configuration du rapport entre la liberté et la soumission au pouvoir. Comme nous l'avons $\mathrm{vu}$, les trois décisions de justice se fondent sur la liberté de s'intégrer dans l'organisation des tours de service et sur la liberté d'accepter les appels de livraison. En somme, dans le travail via une plateforme, " les travailleurs n'ont normalement aucune obligation d'être disposés à exécuter leur prestation d'une manière continue dans le temps ${ }^{21}$. Voilà pourquoi, du point de vue formel, s'il n'y a pas d'obligation de travailler, il ne peut exister de contrat de travail ; le fait qu'un travailleur puisse être obligé de se mettre à disposition ne peut donc permettre de conclure, "sur le fond ${ }^{22}$, que l'on est en présence d'un contrat de travail si la société n'a pas le pouvoir d'exiger, d'organiser et d'utiliser une prestation de travail. En

21 V. De Stefano, "Lavoro "su piattaforma" e lavoro non standard in prospettiva internazionale e comparata », Rivista Giuridica del Lavoro, 2017, I, p. 249 ; P. Loi, « Il lavoro nella gig economy nella prospettiva del rischio », Rivista Giuridica del Lavoro, 2017, I, p. 259.

22 S. Auriemma, "Impresa, lavoro e subordinazione digitale al vaglio della giurisprudenza », Rivista Giuridica del Lavoro, 2017, I, p. 286. 
d'autres termes, le fait d'être disposé à effectuer un travail ne constitue une prestation de travail salarié que si cette disponibilité est formellement obligatoire.

En tout état de cause, ce schéma négocié est connu du droit du travail italien grâce au contrat de travail intermittent régi par le décret-loi $n^{\circ} 81 / 2015$ (semblable au contrat zéro heure au Royaume-Uni ${ }^{23}$ ). En droit italien, dans le cadre d'un contrat de travail intermittent, un travailleur se met à la disposition d'une entreprise qui peut recourir à lui sur appel. La loi italienne impose des contraintes à ce pouvoir d'appel (par exemple un préavis, certaines interdictions d'appel, etc...) qui le rendent inapplicable au travail via la plateforme. Dans le travail intermittent, le temps durant lequel le travailleur se met à la disposition des appels n'a aucune incidence juridique. Cette incidence n'existe que si le travailleur s'engage à répondre à l'appel et, dans ce cas celui-ci aura droit à une indemnité économique correspondant au temps pendant lequel il s'est rendu disponible. Quoi qu'il en soit, même lorsque la disponibilité est obligatoire, le temps de celle-ci ne donne droit à aucune protection juridique, alors qu'à partir du moment où le travailleur répond à l'appel, la prestation de travail est qualifiée de travail subordonné.

Concernant le travail via la plateforme, on prétend que la discontinuité de la prestation de travail représente le contraire de ce qui caractérise le travail subordonné. On estime que le contrat de travail subordonné est continu car, selon la théorie de Coase sur les coûts de transaction, il est plus rationnel pour l'entreprise d'instaurer une seule relation de travail plutôt qu'une multitude. Dans le travail via la plateforme, la rationalité ou la viabilité réside, pour l'entreprise, davantage dans la pluralité successive de contrats que dans un seul contrat continu. Par conséquent, si la continuité est une caractéristique essentielle du travail subordonné, elle ne l'est pas dans le cas du travail via la plateforme ${ }^{24}$.

Cette manière de qualifier les relations de travail confond la nature continue de la prestation (caractéristique du travail subordonné) avec la nature continue de la relation ; cette confusion conduit à considérer également comme une discontinuité de la relation contractuelle la discontinuité de la prestation. Mais tel n'est pas le cas. La discontinuité dans la relation n'exclut pas qu'il puisse y avoir continuité de la prestation ; le contraire étant tout aussi vrai, à savoir que la continuité de la relation n'exclut pas qu'il puisse y avoir discontinuité dans la prestation.

Analyser la dimension temporelle de la prestation de travail via la plateforme consiste à analyser les nouvelles formes de valorisation du capital et, de ce fait, les nouvelles formes du travail salarié comme nouvelles formes de la subordination. Parfois, la prestation de travail via la plateforme n'est pas susceptible d'être utilement mesurée à travers une quantité déterminée de temps calculé à partir des horaires. Dans ces cas-là, l'apport utile pour la valorisation du capital dans le travail cognitif se concrétise dans la dimension « logique » du temps, bien plus que dans sa dimension «chronologique».

Il s'agit d'admettre que l'évolution technique des processus de production (dont la dernière étape est la numérisation) entraîne une perte tendancielle d'utilité du temps de travail envisagé comme un "horaire de travail " prédéterminé, en développant l'utilité

23 A. Adams, M. Freedland, J. Prassl, " "Zero Hours Contracts" in the United Kingdom : Regulating Casula Work, or Legitimating Precarity », Giornale di Diritto del Lavoro e di Relazioni Industriali, 2015, p. 529.

24 P. Ichino, Le conseguenze dell'innovazione tecnologica sul diritto del lavoro, Rivista Italiana di Diritto del Lavoro, 2017, I, p. 525. 
du temps de travail entendu comme un «temps-productif » qui ne tient pas compte du «temps-horaire ». Comme on le voit, le problème du contrat de travail subordonné pose la question de savoir comment configurer la prestation de travail subordonné - c'est-à-dire le temps de travail, le temps qu'une entreprise demande à un travailleur pour valoriser le capital - sans identifier la prestation à la notion ambiguë de travail effectivement effectué, éventuellement mesuré à l'aide d'une montre.

Le travail dans l'économie des plateformes requiert une mise à jour de la dogmatique juridique relative à la notion de "prestation de travail subordonné ", car "les nouveaux visages de la subordination $»^{25}$ sont avant tout les nouveaux visages du temps de travail : le temps de travail (le temps-travail) en tant que temps-productif, celui qui découle d'une obligation contractuelle (temps-contractuel) et qui ne coïncide pas nécessairement avec l'horaire de travail (temps-horaire) ${ }^{26}$. Le travail n'est pas seulement mesuré à travers les horaires parce que l'utilité de la prestation de travail pour l'organisation de la plateforme ne réside pas uniquement dans l'action, mais aussi dans la disponibilité pour l'action.

Cette prise de conscience n'est pas encore acquise dans la jurisprudence italienne, pas même dans les cas examinés ici. Il s'agit là d'une immaturité, qui concerne la théorie générale du contrat de travail sur le rapport logique et dogmatique entre le travail et le temps.

25 A. Supiot, «Les nouveaux visages de la subordination », Droit Social, 2000, p. 131.

26 V. Bavaro, II tempo nel contratto di lavoro subordinato. Critica sulla de-oggettivazione contrattuale del tempo-lavoro, Cacucci, Bari, 2008. En Italie, le concept de "disponibilité continue ", aux fins de de la définition du travail subordonné, est également soutenu par O. Razzolini, "La nozione di subordinazione alla prova delle nuove tecnologie ", Diritto delle Relazioni Industriali, 2014, p. 974 ; S. et F. Siotto, « Fuga dal tempo misurato : il contratto di lavoro tra subordinazione e lavoro immateriale », Rivista Italiana di Diritto del Lavoro, 2010, I, p. 411. 